\title{
Relative Undecidability in Term Rewriting
}

\author{
Alfons Geser ${ }^{1}$, Aart Middeldorp $^{2 \star}$, Enno Ohlebusch ${ }^{3}$, Hans Zantema $^{4 \star \star}$ \\ 1 University of Passau, Germany \\ ${ }^{2}$ University of Tsukuba, Japan \\ 3 University of Bielefeld, Germany \\ 4 Utrecht University, The Netherlands
}

\begin{abstract}
For two hierarchies of properties of term rewriting systems related to confluence and termination, respectively, we prove relative undecidability: for implications $X \Rightarrow Y$ in the hierarchies the property $X$ is undecidable for term rewriting systems satisfying $Y$.
\end{abstract}

\section{Introduction}

In this paper we consider finite term rewriting systems (TRSs) over finite signatures. For these systems termination and confluence are desired properties that are sometimes very hard to prove. Classical results $([7,9])$ state that they are undecidable: no decision procedure exists getting an arbitrary finite TRS as input and giving as output whether the TRS is terminating (confluent) or not. In this paper we don't consider only termination and confluence, but also a number of related properties. For termination they are linearly ordered by implication:

$\mathrm{PT} \Rightarrow \omega \mathrm{T} \Rightarrow \mathrm{TT} \Rightarrow \mathrm{ST} \Rightarrow \mathrm{NSE} \Rightarrow \mathrm{SN} \Rightarrow \mathrm{NL} \Rightarrow \mathrm{AC}$

The acronyms stand for polynomial termination $(\mathrm{PT}), \omega$-termination $(\omega \mathrm{T})$, total termination (TT), simple termination (ST), non-self-embeddingness (NSE), termination (strong normalization, SN), non-loopingness (NL), and acyclicity (AC). We call this the termination hierarchy. One motivation for the properties stronger than termination is that they obey better decomposition theorems. For instance, $\omega$-termination and simple termination satisfy direct sum modularity ([12]), and total termination allows distribution elimination without linearity conditions ([20]). Termination itself does not have these properties. The properties weaker than termination are motivated by frequently occurring shapes of infinite reductions. An extra implication $\mathrm{SN} \Rightarrow \mathrm{WN}$ (weak normalization) can be added as an independent branch in the hierarchy. The confluence hierarchy reads as follows:

$$
\begin{aligned}
& \mathrm{SCR} \Rightarrow \mathrm{CR} \Rightarrow \mathrm{NF} \Rightarrow \mathrm{UN} \Rightarrow \mathrm{UN}^{\rightarrow} \\
& \Downarrow \\
& \text { WCR }
\end{aligned}
$$

\footnotetext{
* Partially supported by the Advanced Information Technology Program (AITP) of the Information Technology Promotion Agency (IPA).

** Email: hansz@cs.ruu.nl.
} 
The acronyms stand for strong confluence (SCR), confluence (or the ChurchRosser property, CR), local confluence (or weak Church-Rosser, WCR), the normal form property (NF), unique normal forms (UN), and unique normal forms with respect to reduction $\mathrm{UN}^{\rightarrow}$. For weakly normalizing systems the properties $\mathrm{CR}, \mathrm{NF}, \mathrm{UN}$, and $\mathrm{UN}^{\rightarrow}$ coincide. For terminating systems also WCR and CR coincide and are decidable.

Undecidability of confluence is well-known ([9]), for the other properties in the confluence hierarchy it is easy to see too. Also undecidability of most of the properties related to termination is known ([7, 17, 1, 15, 22]), sometimes even for single rules $([2,15,13])$. The undecidability of $\omega$-termination is a new result; this paper includes a sketch of the proof.

In this paper we do not only provide a general framework for proving this kind of undecidability, for all implications in the hierarchies except one- $\mathrm{PT} \Rightarrow \omega \mathrm{T}-$ we prove the stronger result of relative undecidability: for such an implication $X \Rightarrow Y$ we prove that the property $X$ is undecidable for TRSs satisfying $Y$. As a consequence, relative undecidability of $X \Rightarrow Z$ immediately follows from validity of the implication $Y \Rightarrow Z$ and relative undecidability of $X \Rightarrow Y$.

All of our proofs are given by means of Post's Correspondence Problem (PCP) in the following way: for all of the implications $X \Rightarrow Y$ and all instances of PCP we construct a TRS that always satisfies $Y$, and either satisfies $X$ if and only if the PCP instance admits a solution, or satisfies $X$ if and only if the PCP instance admits no solution. Since PCP is known to be undecidable $([18])$, this proves relative undecidability of the implication $X \Rightarrow Y$.

The main part of the paper consists of constructions of such TRSs parametrized by PCP instances and corresponding proofs of the above mentioned properties. In the next section this is done for the confluence hierarchy and in Sect. 3 for the termination hierarchy. These two sections can be read independently. The definitions of the various properties are given in the respective sections; for further preliminaries on term rewriting we refer to $[3,10]$. In the remainder of this section we recall the formulation of PCP:

given a finite alphabet $\Gamma$ and a finite set $P \subset \Gamma^{+} \times \Gamma^{+}$, is there some natural number $n>0$ and $\left(\alpha_{i}, \beta_{i}\right) \in P$ for $i=1, \ldots, n$ such that $\alpha_{1} \alpha_{2} \cdots \alpha_{n}=\beta_{1} \beta_{2} \cdots \beta_{n}$ ?

The set $P$ is called an instance of PCP, the string $\alpha_{1} \alpha_{2} \cdots \alpha_{n}=\beta_{1} \beta_{2} \cdots \beta_{n}$ a solution for $P$. Matiyasevich and Senizergues [14] recently showed that PCP is undecidable even when restricted to instances consisting of seven pairs. We assume that $\Gamma$ is fixed throughout the paper. (One may assume that $\Gamma=\{0,1\}$.) In our TRSs we need for every $a \in \Gamma$ a unary symbol $a$ (and sometimes also unary symbols $\bar{a}, \hat{a}$ and $\tilde{a})$. For any string $\alpha=a_{1} a_{2} \cdots a_{n} \in \Gamma^{*}$ and any term $t$ we define $\alpha(t)=a_{1}\left(a_{2}\left(\cdots\left(a_{n}(t)\right) \cdots\right)\right)$.

\section{The Confluence Hierarchy}

In this section we show relative undecidability of all implications in the confluence hierarchy as presented in the introduction. Actually we show the stronger 
result that relative undecidability holds for linear TRSs.

Let us first recall the definitions of the six properties properties in the confluence hierarchy. A TRS $\mathcal{R}$ is called confluent (or Church-Rosser, CR) if $\leftarrow_{\mathcal{R}}^{*} \cdot \rightarrow_{\mathcal{R}}^{*} \subseteq \rightarrow_{\mathcal{R}}^{*} \cdot \leftarrow_{\mathcal{R}}^{*}$, or, equivalently, every two convertible terms have a common reduct. A TRS $\mathcal{R}$ is called locally confluent (or weakly Church-Rosser, WCR) if $\leftarrow_{\mathcal{R}} \cdot \rightarrow_{\mathcal{R}} \subseteq \rightarrow_{\mathcal{R}}^{*} \cdot \leftarrow_{\mathcal{R}}^{*}$. A TRS $\mathcal{R}$ is called strongly confluent (or strongly Church-Rosser, SCR) if $\leftarrow_{\mathcal{R}} \cdot \rightarrow_{\mathcal{R}} \subseteq \rightarrow_{\overline{\mathcal{R}}} \cdot \leftarrow_{\mathcal{R}}^{*}$. A TRS $\mathcal{R}$ is said to have the normal form property (NF) if every term convertible to a normal form reduces to that normal form, or, equivalently, every term that has a normal form is confluent. A TRS $\mathcal{R}$ is said to have unique normal forms (UN) if different normal forms are not convertible. A TRS $\mathcal{R}$ is said to have unique normal forms with respect to reduction ( $\mathrm{UN}^{\rightarrow}$ ) if every term has at most one normal form. The above definition of strong confluence originates from Huet [8] and is different from the one in Dershowitz and Jouannaud [3]. They call a TRS $\mathcal{R}$ strongly confluent if $\leftarrow_{\mathcal{R}} \cdot \rightarrow_{\mathcal{R}} \subseteq \rightarrow \overline{\overline{\mathcal{R}}} \cdot \leftarrow \overline{\overline{\mathcal{R}}}$. Klop [10] calls the latter property subcommutativity $\left(\mathrm{WCR}^{\leq 1}\right)$.

Below we use PCP to show that for each of the five implications $X \Rightarrow Y$ in the confluence hierarchy the property $X$ is undecidable for TRSs satisfying the property $Y$. A key observation is that an arbitrary PCP instance $P$ admits a solution if and only if $A \rightarrow_{\mathcal{R}_{0}(P)}^{*} B$ for the TRS

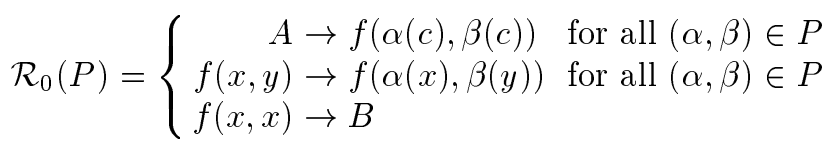

To arrive at results for linear TRSs and for some technical convenience this basic system is replaced by

$$
\mathcal{R}_{1}(P)=\left\{\begin{array}{rlrl}
A & \rightarrow f(\alpha(c), \beta(c)) & & \text { for all }(\alpha, \beta) \in P \\
f(x, y) & \rightarrow f(\alpha(x), \beta(y)) & \text { for all }(\alpha, \beta) \in P \\
f(x, y) & \rightarrow g(x, y) & & \\
f(x, y) & \rightarrow A & & \\
g(x, y) & \rightarrow A & & \text { for all } a \in \Gamma \\
g(a(x), a(y)) & \rightarrow g(x, y) & & \\
g(c, c) & \rightarrow B & &
\end{array}\right.
$$

Proposition 1. $A \rightarrow_{\mathcal{R}_{1}(P)}^{*} B$ if and only if $P$ admits a solution.

Proof. Suppose $\gamma \in \Gamma^{+}$is a solution for $P$. So $\gamma=\alpha_{1} \cdots \alpha_{n}=\beta_{1} \cdots \beta_{n}$ for some $n \geq 1$ with $\left(\alpha_{i}, \beta_{i}\right) \in P$ for $i=1, \ldots, n$. We have the following reduction in $\mathcal{R}_{1}(P)$ :

$$
\begin{aligned}
A & \rightarrow f\left(\alpha_{n}(c), \beta_{n}(c)\right) \rightarrow^{*} f\left(\alpha_{1} \cdots \alpha_{n}(c), \beta_{1} \cdots \beta_{n}(c)\right)=f(\gamma(c), \gamma(c)) \\
& \rightarrow g(\gamma(c), \gamma(c)) \rightarrow^{+} g(c, c) \rightarrow B .
\end{aligned}
$$

Conversely, suppose that $A \rightarrow_{\mathcal{R}_{1}(P)}^{*} B$. Beyond the last $A$ occurring in this 
reduction sequence it is of the form

$$
\begin{aligned}
A & \rightarrow f\left(\alpha_{n}(c), \beta_{n}(c)\right) \rightarrow^{*} f\left(\alpha_{1} \cdots \alpha_{n}(c), \beta_{1} \cdots \beta_{n}(c)\right) \\
& \rightarrow \underbrace{g\left(\alpha_{1} \cdots \alpha_{n}(c), \beta_{1} \cdots \beta_{n}(c)\right) \rightarrow^{*} g(c, c)} \rightarrow B .
\end{aligned}
$$

for some $n \geq 1$ with $\left(\alpha_{i}, \beta_{i}\right) \in P$ for $i=1, \ldots, n$. In the underbraced part only rewrite rules of the form $g(a(x), a(y)) \rightarrow g(x, y)$ are used. Hence $\alpha_{1} \cdots \alpha_{n}(c)=$ $\beta_{1} \cdots \beta_{n}(c)$, giving a solution for $P$.

Below we make frequent use of the following result of Huet [8].

Theorem 2. Every linear strongly closed TRS is strongly confluent.

Here a TRS $\mathcal{R}$ is called strongly closed if both $s \rightarrow \overline{\overline{\mathcal{R}}} \cdot \leftarrow_{\mathcal{R}}^{*} t$ and $t \rightarrow \overline{\overline{\mathcal{R}}} \cdot \leftarrow_{\mathcal{R}}^{*} s$ for every critical pair $\langle s, t\rangle$ of $\mathcal{R}$.

\section{$2.1 \quad \mathrm{NF} \Rightarrow \mathrm{UN}$}

Proposition 3. The TRS $\mathcal{R}_{1}(P)$ has unique normal forms for every PCP instance $P$.

Proof. Consider the TRS $\mathcal{R}_{1}^{\prime}(P)=\mathcal{R}_{1}(P) \cup\{A \rightarrow B, f(x, y) \rightarrow B, g(x, y) \rightarrow B\}$. The relations $\leftrightarrow_{\mathcal{R}_{1}(P)}^{*}$ and $\leftrightarrow_{\mathcal{R}_{1}^{\prime}(P)}^{*}$ clearly coincide. Also the normal forms of the two TRSs are the same. The TRS $\mathcal{R}_{1}^{\prime}(P)$ is linear and strongly closed hence (strongly) confluent by Thm. 2. This implies that $\mathcal{R}_{1}(P)$ has unique normal forms.

Proposition 4. The following statements are equivalent:

1. The TRS $\mathcal{R}_{1}(P)$ has the normal form property.

2. The TRS $\mathcal{R}_{1}(P)$ is confluent.

3. The PCP instance $P$ admits a solution.

Proof. Since confluence implies the normal form property, according to Prop. 1 it suffices to show that (i) $A \rightarrow_{\mathcal{R}_{1}(P)}^{*} B$ whenever $\mathcal{R}_{1}(P)$ has the normal form property and (ii) $\mathcal{R}_{1}(P)$ is confluent whenever $A \rightarrow_{\mathcal{R}_{1}(P)}^{*} B$. For (i) we note that $A \leftarrow g(c, c) \rightarrow B$ in $\mathcal{R}_{1}(P)$ with $B$ a normal form, hence $A \rightarrow_{\mathcal{R}_{1}(P)}^{*} B$ by definition of the normal form property. For (ii) we consider the confluent TRS $\mathcal{R}_{1}^{\prime}(P)$ defined in the proof of Prop. 3. From $A \rightarrow_{\mathcal{R}_{1}(P)}^{*} B$ we obtain that the relations $\rightarrow_{\mathcal{R}_{1}(P)}^{*}$ and $\rightarrow_{\mathcal{R}_{1}^{\prime}(P)}^{*}$ coincide. Hence $\mathcal{R}_{1}(P)$ is confluent too. 


\section{$2.2 \mathrm{CR} \Rightarrow \mathrm{NF}$}

Let $\mathcal{R}_{2}(P)=\mathcal{R}_{1}(P) \cup\{B \rightarrow B\}$.

Proposition 5. The TRS $\mathcal{R}_{2}(P)$ has the normal form property for every PCP instance $P$.

Proof. The set of normal forms of $\mathcal{R}_{2}(P)$ coincides with the set of weakly normalizing terms. Hence the normal form property is trivially satisfied.

Proposition 6. The TRS $\mathcal{R}_{2}(P)$ is confluent if and only if $P$ admits a solution.

Proof. Since the relations $\rightarrow_{\mathcal{R}_{2}(P)}^{*}$ and $\rightarrow_{\mathcal{R}_{1}(P)}^{*}$ coincide, $\mathcal{R}_{2}(P)$ is confluent if and only if $\mathcal{R}_{1}(P)$ is confluent. Hence the result follows from Prop. 4.

\section{$2.3 \quad \mathrm{SCR} \Rightarrow \mathrm{CR}$}

Let $\mathcal{R}_{3}(P)=\mathcal{R}_{1}(P) \cup\{B \rightarrow C, C \rightarrow A\}$.

Proposition 7. The TRS $\mathcal{R}_{3}(P)$ is confluent for every PCP instance $P$.

Proof. One easily checks that the linear TRS $\mathcal{R}_{3}^{\prime}(P)=\mathcal{R}_{3}(P) \cup\{B \rightarrow A\}$ is strongly closed hence (strongly) confluent by Thm. 2. Since the relations $\rightarrow_{\mathcal{R}_{3}(P)}^{*}$ and $\rightarrow_{\mathcal{R}_{3}^{\prime}(P)}^{*}$ coincide, $\mathcal{R}_{3}(P)$ is also confluent.

Proposition 8. The TRS $\mathcal{R}_{3}(P)$ is strongly confluent if and only if $P$ admits a solution.

Proof. In a shortest $\mathcal{R}_{3}(P)$-reduction sequence from $A$ to $B$ the rewrite rules $B \rightarrow C$ and $C \rightarrow A$ are not used. Hence $A \rightarrow_{\mathcal{R}_{3}(P)}^{*} B$ if and only if $A \rightarrow_{\mathcal{R}_{1}(P)}^{*} B$. According to Prop. 1 we have to show that $\mathcal{R}_{3}(P)$ is strongly confluent if and only if $A \rightarrow_{\mathcal{R}_{3}(P)}^{*} B$. In $\mathcal{R}_{3}(P)$ we have $B \leftarrow g(c, c) \rightarrow A$. If $\mathcal{R}_{3}(P)$ is strongly confluent then $B \rightarrow^{=} \cdot \leftarrow^{*} A$, so either $B \leftarrow^{*} A$ or $B \rightarrow C \leftarrow^{*} A$. Since any reduction sequence from $A$ to $C$ must pass through $B$, in both cases we have the desired $A \rightarrow_{\mathcal{R}_{3}(P)}^{*} B$. Conversely, if $A \rightarrow_{\mathcal{R}_{3}(P)}^{*} B$ then one easily checks that $\mathcal{R}_{3}(P)$ is strongly closed and therefore strongly confluent by Thm. 2 .

\section{$2.4 \mathrm{CR} \Rightarrow \mathrm{WCR}$}

Let $\mathcal{R}_{4}(P)=\mathcal{R}_{1}(P) \cup\{B \rightarrow f(c, c), B \rightarrow C\}$.

Proposition 9. The TRS $\mathcal{R}_{4}(P)$ is locally confluent for every PCP instance P.

Proof. One easily checks that all critical pairs of $\mathcal{R}_{4}(P)$ are joinable.

Proposition 10. The TRS $\mathcal{R}_{4}(P)$ is confluent if and only if $P$ admits a solution. 
Proof. In a shortest $\mathcal{R}_{4}(P)$-reduction sequence from $A$ to $B$ the rewrite rules $B \rightarrow f(c, c)$ and $B \rightarrow C$ are not used. Hence $A \rightarrow_{\mathcal{R}_{4}(P)}^{*} B$ if and only if $A \rightarrow \mathcal{R}_{\mathcal{R}_{1}(P)}^{*} B$. According to Prop. 1 we have to show that $\mathcal{R}_{4}(P)$ is confluent if and only if $A \rightarrow_{\mathcal{R}_{4}(P)}^{*} B$. In $\mathcal{R}_{4}(P)$ we have $A \leftarrow f(c, c) \leftarrow B \rightarrow C$. If $\mathcal{R}_{4}(P)$ is confluent then $A \rightarrow_{\mathcal{R}_{4}(P)}^{*} C$ which is equivalent to $A \rightarrow_{\mathcal{R}_{4}(P)}^{*} B$. Conversely, if $A \rightarrow_{\mathcal{R}_{4}(P)}^{*} B$ then we obtain confluence by considering the linear and strongly closed TRS $\mathcal{R}_{4}^{\prime}(P)=\mathcal{R}_{4}(P) \cup\{A \rightarrow C, f(x, y) \rightarrow C, g(x, y) \rightarrow C\}$.

\section{$2.5 \mathrm{UN} \Rightarrow \mathrm{UN} \rightarrow$}

In this subsection we assume that PCP instances are presented as ordered lists $\left(\alpha_{1}, \beta_{1}\right),\left(\alpha_{2}, \beta_{2}\right), \ldots,\left(\alpha_{n}, \beta_{n}\right)$ rather than sets. This entails no loss of generality. Let $\mathcal{R}_{5}(P)$ be the union of $\mathcal{R}_{5}^{1}=\{f(c, c, c, w) \rightarrow A\}$,

$$
\mathcal{R}_{5}^{2}(P)=\left\{\begin{aligned}
f(x, y, z, i(w)) & \rightarrow f\left(\alpha_{i}(x), \beta_{i}(y), i(z), w\right) & & \text { for all } i \in\{1, \ldots, n\} \\
f(x, y, i(z), c) & \rightarrow g(x, y, i(z)) & & \text { for all } i \in\{1, \ldots, n\} \\
g(x, y, i(z)) & \rightarrow g(x, y, z) & & \text { for all } i \in\{1, \ldots, n\} \\
g(a(x), a(y), c)) & \rightarrow g(x, y, c) & & \text { for all } a \in \Gamma \\
g(c, c, c) & \rightarrow B & &
\end{aligned}\right.
$$

and $\{f(x, y, z, w) \rightarrow f(x, y, z, w), g(x, y, z) \rightarrow g(x, y, z), A \rightarrow A, C \rightarrow A, C \rightarrow$ $D\}$. Note that $n$ depends on the PCP instance $P$; for every $i \in\{1, \ldots, n\}$ we have a unary function symbol $i$.

Proposition 11. $A \leftrightarrow_{\mathcal{R}_{5}(P)}^{*} B$ if and only if $P$ admits a solution.

Proof. Abbreviate $\mathcal{R}_{5}^{1} \cup \mathcal{R}_{5}^{2}(P)$ to $\mathcal{R}_{5}^{\prime}(P)$. Suppose $\gamma \in \Gamma^{+}$is a solution for $P$. So $\gamma=\alpha_{i_{1}} \cdots \alpha_{i_{m}}=\beta_{i_{1}} \cdots \beta_{i_{m}}$ for some $m \geq 1$ and $i_{1}, \ldots, i_{m} \in\{1, \ldots, n\}$. Let $t=i_{m} \cdots i_{1}(c)$ and $t^{\prime}=i_{1} \cdots i_{m}(c)$. We have the following conversion in $\mathcal{R}_{5}^{\prime}(P)$ :

$$
\begin{aligned}
A & \leftarrow f(c, c, c, t) \rightarrow^{*} f\left(\gamma(c), \gamma(c), t^{\prime}, c\right) \rightarrow g\left(\gamma(c), \gamma(c), t^{\prime}\right) \rightarrow^{*} g(\gamma(c), \gamma(c), c) \\
& \rightarrow^{*} g(c, c, c) \rightarrow B .
\end{aligned}
$$

Conversely suppose that $A$ and $B$ are convertible in $\mathcal{R}_{5}(P)$. Consider a shortest conversion between $A$ and $B$. Since the rewrite rules in $\mathcal{R}_{5}(P) \backslash \mathcal{R}_{5}^{\prime}(P)$ don't contribute to a shortest conversion between $A$ and $B$, it must be of the form $A \leftarrow f(c, c, c, t) \leftrightarrow_{\mathcal{R}_{5}^{\prime}(P)}^{*} B$ for some term $t$ such that in the conversion between $f(c, c, c, t)$ and $B$ no $\mathcal{R}_{5}^{1}$-steps take place at root positions. Using the fact that $\mathcal{R}_{5}^{2}(P)$ is linear and non-erasing one easily concludes that there are no $\mathcal{R}_{5}^{1}$ steps in the conversion between $f(c, c, c, t)$ and $B$. Hence $f(c, c, c, t)$ and $B$ are convertible in $\mathcal{R}_{5}^{2}(P)$. Because the TRS $\mathcal{R}_{5}^{2}(P)$ is orthogonal hence confluent and $B$ is a normal form, we obtain $f(c, c, c, t) \rightarrow_{\mathcal{R}_{5}^{2}(P)}^{*} B$. Now it easy to show that the term $t$ codes a solution for $P$.

The sole purpose of the rules $f(x, y, z, w) \rightarrow f(x, y, z, w)$ and $g(x, y, z) \rightarrow$ $g(x, y, z)$ is to avoid unwanted normal forms in $\mathcal{R}_{5}(P)$, whose presence would considerably complicate the proofs of Props. 12 and 13 below. 
Note that the above proposition doesn't hold for (the TRSs based on) $\mathcal{R}_{1}(P)$ because in $\mathcal{R}_{1}(P)$ the terms $A$ and $B$ may be convertible even if $P$ admits no solution. For instance, we have $A \rightarrow f(100(c), 10(c)) \leftarrow f(0(c), 0(c)) \rightarrow^{*} B$ in $\mathcal{R}_{1}(\{(100,10),(10,1)\})$.

Proposition 12. The TRS $\mathcal{R}_{5}(P)$ has unique normal forms with respect to reduction for every $P C P$ instance $P$.

Proof. By induction on the structure of terms we can easily prove that every term has at most one normal form.

Proposition 13. The TRS $\mathcal{R}_{5}(P)$ has unique normal forms if and only if $P$ does not have a solution.

Proof. According to Prop. 11 we have to show that $\mathcal{R}_{5}(P)$ admits two different convertible normal forms if and only if $A$ and $B$ are convertible. If $A$ and $B$ are convertible then so are the different normal forms $B$ and $D: B \leftrightarrow{ }^{*} A \leftarrow C \rightarrow D$. Conversely, suppose that $\mathcal{R}_{5}(P)$ admits two different convertible normal forms $t_{1}, t_{2}$. Then we can write $t_{i}=\alpha_{i}\left(s_{i}\right)$ with $\alpha_{i} \in(\Gamma \cup\{1, \ldots, n\})^{*}$ and $s_{i}$ is either $B, D, c$ or a variable, for $i=1,2$. Due to the shape of the rules $\alpha_{1}$ is not affected in the conversion $\alpha_{1}\left(s_{1}\right) \leftrightarrow^{*} \alpha_{2}\left(s_{2}\right)$, hence $\alpha_{1}=\alpha_{2}$ and $s_{1} \leftrightarrow^{*} s_{2}$, where $s_{1}$ and $s_{2}$ are different. Since no non-trivial conversion is possible starting from $c$ or a variable, we conclude that $s_{1}$ and $s_{2}$ are $D$ and $B$, or vice versa. Hence $D$ and $B$ are convertible, and since $A \leftarrow C \rightarrow D$ also $A$ and $B$ are convertible.

\section{The Termination Hierarchy}

In this section we show relative undecidability of the last six implications in the termination hierarchy as presented in the introduction.

Before we can define the properties in the termination hierarchy, we need a few preliminary definitions. Throughout the following we assume that $\mathcal{F}$ is a finite signature containing at least one constant. A (strict partial) order $>$ on the set $\mathcal{T}(\mathcal{F})$ of ground terms is called monotonic if for all $f \in \mathcal{F}$ and $t, u \in \mathcal{T}(\mathcal{F})$ with $t>u$ we have $f(\ldots, t, \ldots)>f(\ldots, u, \ldots)$. A TRS $\mathcal{R}$ over $\mathcal{F}$ and an order $>$ on $\mathcal{T}(\mathcal{F})$ are called compatible if $t>u$ for all rewrite steps $t \rightarrow_{\mathcal{R}} u$. For compatibility with a monotonic order it suffices to check that $l \sigma>r \sigma$ for all rules $l \rightarrow r$ in $\mathcal{R}$ and all ground substitutions $\sigma$. An $\mathcal{F}$-algebra consists of a set $A$ and for every $f \in \mathcal{F}$ a function $f_{A}: A^{n} \rightarrow A$, where $n$ is the arity of f. A monotone $\mathcal{F}$-algebra $(A,>)$ is an $\mathcal{F}$-algebra $A$ for which the underlying set is provided with an order $>$ such that every algebra operation is monotonic in all of its arguments. More precisely, for all $f \in \mathcal{F}$ and $a, b \in A$ with $a>b$ we have $f_{A}(\ldots, a, \ldots)>f_{A}(\ldots, b, \ldots)$. A monotone $\mathcal{F}$-algebra $(A,>)$ is called well-founded if $>$ is a well-founded order. A monotone $\mathcal{F}$-algebra $(A,>)$ is called simple if for all $n$-ary $f \in \mathcal{F}$ with $n \geq 1, a_{1}, \ldots, a_{n} \in A$, and $i=1, \ldots, n$ we have $f_{A}\left(a_{1}, \ldots, a_{n}\right) \geq a_{i}$. Every monotone $\mathcal{F}$-algebra $(A,>)$ induces an order $>_{A}$ on the set of terms $\mathcal{T}(\mathcal{F}, \mathcal{X})$ as follows: $t>_{A} u$ if and only if $[\alpha](t)>[\alpha](u)$ 
for all assignments $\alpha: \mathcal{X} \rightarrow A$. Here $[\alpha]$ denotes the homomorphic extension of $\alpha$, i.e., $[\alpha](x)=\alpha(x)$ and $[\alpha]\left(f\left(t_{1}, \ldots, t_{n}\right)\right)=f_{A}\left([\alpha]\left(t_{1}\right), \ldots,[\alpha]\left(t_{n}\right)\right)$ for $x \in \mathcal{X}$, $f \in \mathcal{F}$, and $t_{1}, \ldots, t_{n} \in \mathcal{T}(\mathcal{F}, \mathcal{X})$. For ground terms $t$ the value $[\alpha](t)$ does not depend on $\alpha$ and is simply written as $[t]$. A TRS $\mathcal{R}$ and a monotone algebra $(A,>)$ are called compatible if $\mathcal{R}$ and $>_{A}$ are compatible. The set of rewrite rules $f\left(x_{1}, \ldots, x_{n}\right) \rightarrow x_{i}$ for all $f \in \mathcal{F}$ and all $i=1, \ldots, n$, where $n \geq 1$ is the arity of $f$, is denoted by $\mathcal{E} \operatorname{mb}(\mathcal{F})$, or simply by $\mathcal{E}$ mb when the signature $\mathcal{F}$ can be inferred from the context.

The properties in the termination hierarchy are now defined as follows. A TRS is called terminating (or strongly normalizing, SN) if it does not allow infinite reductions, or, equivalently, it is compatible with a well-founded monotone algebra. A TRS $\mathcal{R}$ is called weakly normalizing (WN) if every term reduces to at least one normal form. A TRS $\mathcal{R}$ over a signature $\mathcal{F}$ is called simply terminating if $\mathcal{R} \cup \mathcal{E} \operatorname{mb}(\mathcal{F})$ is terminating, or, equivalently, it is compatible with a simple monotone $\mathcal{F}$-algebra. A TRS over a signature $\mathcal{F}$ is called totally terminating if it is compatible with a monotonic well-founded total order on $\mathcal{T}(\mathcal{F})$, or, equivalently, it is compatible with a well-founded monotone $\mathcal{F}$-algebra $(A,>)$ in which the order $>$ is total. A TRS over a signature $\mathcal{F}$ is called $\omega$-terminating if it is compatible with a well-founded monotone $\mathcal{F}$-algebra $(A,>)$ in which $A=\mathbb{N}$ and $>$ is the usual order on $\mathbb{N}$. A TRS over a signature $\mathcal{F}$ is called polynomially terminating if it is compatible with a well-founded monotone $\mathcal{F}$-algebra $(A,>)$ in which $A=\mathbb{N},>$ is the usual order on $\mathbb{N}$, and for which all functions $f_{A}$ are polynomials. A TRS $\mathcal{R}$ is called looping if it admits a reduction $t \rightarrow \rightarrow_{\mathcal{R}}^{+} C[t \sigma]$ for some term $t$, context $C$, and substitution $\sigma$. A TRS $\mathcal{R}$ is called cyclic if it admits a reduction $t \rightarrow \rightarrow_{\mathcal{R}}^{+} t$ for some term $t$. A TRS $\mathcal{R}$ is called self-embedding if it admits a reduction $t \rightarrow_{\mathcal{R}}^{+} u \rightarrow_{\mathcal{E} \mathrm{mb}}^{*} t$ for some terms $t, u$.

Recent investigations of these notions include [4, 5, 16, 19, 20, 23]. Validity of most of the implications in the termination hierarchy is direct from the definitions; only TT $\Rightarrow$ ST requires some well-known argument, see e.g. [20], and NSE $\Rightarrow$ SN requires Kruskal's theorem. None of the implications are equivalences: for all implications $X \Rightarrow Y$ in the termination hierarchy a TRS exists satisfying $Y$ but not $X$. For infinite TRSs over infinite signatures the termination hierarchy is more complicated: if the notion of embedding is not changed then NSE $\Rightarrow \mathrm{SN}$ does not hold any more, if the notions of embedding and simple termination are adjusted as motivated in [16], then the implication TT $\Rightarrow$ ST no longer holds ([16]). In this paper however we consider only finite TRSs over finite signatures.

All TRSs needed for the termination hierarchy are modifications of two basic TRSs parameterized by an arbitrary PCP instance $P$. For any string $\alpha=a_{1} a_{2} \ldots a_{n} \in \Gamma^{*}$ and any term $t$ we define $\bar{\alpha}(t)=\bar{a}_{n}\left(\cdots\left(\bar{a}_{2}\left(\bar{a}_{1}(t)\right)\right) \cdots\right)$. The two basic TRSs are

$$
\begin{aligned}
& \mathcal{R}(P)=\left\{\begin{array}{cl}
F(c, c, a(z)) \rightarrow F(a(z), a(z), a(z)) & \text { for all } a \in \Gamma \\
F(\alpha(x), \beta(y), z) \rightarrow F(x, y, z) & \text { for all }(\alpha, \beta) \in P
\end{array}\right. \\
& \mathcal{S}(P)=\left\{\begin{array}{cl}
F(x, \bar{a}(y), x, \bar{a}(y)) \rightarrow F(a(x), y, a(x), y) & \text { for all } a \in \Gamma \\
F(\alpha(x), y, \beta(z), w) \rightarrow F(x, \bar{\alpha}(y), z, \bar{\beta}(w)) & \text { for all }(\alpha, \beta) \in P
\end{array}\right.
\end{aligned}
$$

The system $\mathcal{R}(P)$ is a minor modification of the basic system from [13]; the 
system $\mathcal{S}(P)$ is from [22]. The next well-known proposition is the motivation for defining these systems.

Proposition 14. The following statements are equivalent:

1. The $\operatorname{TR} S \mathcal{R}(P)$ is terminating.

2. The $T R S \mathcal{S}(P)$ is terminating.

3. The $P C P$ instance $P$ admits no solution.

Proof. We sketch the proof of the equivalence of 1 and 3 ; the equivalence proof of 2 and 3 is similar. Suppose $\gamma \in \Gamma^{+}$is a solution for $P$. So $\gamma=\alpha_{1} \cdots \alpha_{n}=$ $\beta_{1} \cdots \beta_{n}$ for some $n \geq 1$ with $\left(\alpha_{i}, \beta_{i}\right) \in P$ for $i=1, \ldots, n$. We have the following cyclic reduction in $\mathcal{R}(P)$ :

$$
\begin{aligned}
F(\gamma(c), \gamma(c), \gamma(c)) & \rightarrow F\left(\alpha_{2} \cdots \alpha_{n}(c), \beta_{2} \cdots \beta_{n}(c), \gamma(c)\right) \rightarrow^{*} F(c, c, \gamma(c)) \\
& \rightarrow F(\gamma(c), \gamma(c), \gamma(c)) .
\end{aligned}
$$

Conversely, suppose that $\mathcal{R}(P)$ admits an infinite reduction. It is not difficult to see that there exists an infinite reduction in which all steps take place at the root position and both kinds of rewrite rules are used infinitely often. (This can be shown formally using type elimination [20].) Any such reduction must contain a subsequence of the form

$$
F(c, c, a(t)) \rightarrow \underbrace{\left.F(a(t), a(t), a(t)) \rightarrow^{+} F(c, c, a(t))\right)}
$$

where in the underbraced part only rewrite rules of the form $F(\alpha(x), \beta(y), z) \rightarrow$ $F(x, y, z)$ are used. Hence $a(t)=\alpha_{1} \cdots \alpha_{n}(c)=\beta_{1} \cdots \beta_{n}(c)$ for some $n \geq 1$ with $\left.\alpha_{i}, \beta_{i}\right) \in P$ for $i=1, \ldots, n$, giving a solution for $P$.

This proves undecidability of termination. Since the constructed infinite reduction is always cyclic, this also proves undecidability of both loopingness and cyclicity. The advantage of $\mathcal{S}(P)$ over $\mathcal{R}(P)$ is that it is length-preserving, which means that $|l \sigma|=|r \sigma|$ for all rules $l \rightarrow r$ in $\mathcal{S}(P)$ and all ground substitutions $\sigma$. Here $|t|$ denotes the number of function symbols in $t$. Since for length-preserving TRSs termination and simple termination coincide, this proves that both simple termination and self-embeddingness are undecidable. The main result of [22] is that $\mathcal{S}(P)$ is totally terminating if and only if $P$ admits no solution, proving undecidability of total termination.

\section{$3.1 \quad \mathrm{NL} \Rightarrow \mathrm{AC}$}

Let

$$
\mathcal{S}_{1}(P)=\left\{\begin{aligned}
F(c, c, a(z)) & \rightarrow g(F(a(z), a(z), a(z))) & & \text { for all } a \in \Gamma \\
F(\alpha(x), \beta(y), z) & \rightarrow F(x, y, z) & & \text { for all }(\alpha, \beta) \in P
\end{aligned}\right.
$$

Proposition 15. The TRS $\mathcal{S}_{1}(P)$ is acyclic for every PCP instance $P$. 
Proof. For a proof by contradiction, assume a cyclic $\mathcal{S}_{1}(P)$-reduction $t \rightarrow^{+} t$ exists. Applying rules of the form $F(c, c, a(z)) \rightarrow g(F(a(z), a(z), a(z)))$ strictly increases the number of $g$ symbols, while the other kind of rules does not change the number of $g$ symbols. Hence in a reduction $t \rightarrow^{+} t$ only rules of the second kind are applied. But these rules constitute a terminating system, yielding the desired contradiction.

Proposition 16. The $T R S \mathcal{S}_{1}(P)$ is looping if and only if $P$ admits a solution.

Proof. If $\gamma \in \Gamma^{+}$is a solution for $P$ then we have the $\mathcal{S}_{1}(P)$-loop

$$
F(\gamma(c), \gamma(c), \gamma(c)) \rightarrow^{+} F(c, c, \gamma(c)) \rightarrow g(F(\gamma(c), \gamma(c), \gamma(c)))
$$

Conversely, if $\mathcal{S}_{1}(P)$ is looping then it admits an infinite reduction. Erasing all occurrences of $g$ in any infinite $\mathcal{S}_{1}(P)$-reduction yields an infinite $\mathcal{R}(P)$ reduction. According to Prop. 14, $P$ admits a solution.

\section{$3.2 \mathrm{SN} \Rightarrow \mathrm{NL}$}

Let

$$
\mathcal{S}_{2}(P)=\left\{\begin{aligned}
h(F(c, c, a(z))) & \rightarrow g(F(a(z), a(z), a(z))) & & \text { for all } a \in \Gamma \\
F(\alpha(x), \beta(y), z) & \rightarrow F(x, y, z) & & \text { for all }(\alpha, \beta) \in P \\
h(g(x)) & \rightarrow g(h(x)) & & \\
f(g(x)) & \rightarrow f(h(h(x))) & &
\end{aligned}\right.
$$

Proposition 17. The TRS $\mathcal{S}_{2}(P)$ is non-looping for every PCP instance $P$.

Proof. For arbitrary terms $t$ define inductively:

$$
\begin{aligned}
& \phi(x)=0 \\
& \phi(c)=0 \\
& \phi(a(t))=0 \\
& \phi(f(t))=0 \\
& \phi(g(t))=1+\phi(t) \\
& \phi(h(t))=1+\phi(t) \\
& \phi\left(F\left(t_{1}, t_{2}, t_{3}\right)\right)=0 \\
& \psi(x)=0 \\
& \psi(c)=0 \\
& \text { for } x \in \mathcal{X} \\
& \psi(a(t))=1+\psi(t) \\
& \text { for } a \in \Gamma \\
& \psi(f(t))=1+\psi(t) \\
& \psi(g(t))=\psi(t) \\
& \psi(h(t))=\psi(t) \\
& \psi\left(F\left(t_{1}, t_{2}, t_{3}\right)\right)=1+\max \left\{\psi\left(t_{1}\right), \psi\left(t_{2}\right), \psi\left(t_{3}\right)\right\}
\end{aligned}
$$

For every $\mathcal{S}_{2}(P)$-reduction step $t \rightarrow u$ we have $\phi(t)=\phi(u)$ and $\psi(t) \geq \psi(u)$. Assume $\mathcal{S}_{2}(P)$ admits a loop. Choose a loop $t \rightarrow^{+} C[t \sigma]$ for which the nesting of $F$ symbols in $t$ is minimal. From $\psi(t) \geq \psi(C[t \sigma])$ we conclude that $C$ only consists of $g$ and $h$ symbols; from $\phi(t)=\phi(C[t \sigma])$ we conclude that $C$ is the trivial context. Hence $t \rightarrow^{+} t \sigma$. Write $t=D\left[F\left(t_{1}, t_{2}, t_{3}\right)\right]$ for $D$ consisting only of unary symbols. Hence $D\left[F\left(t_{1}, t_{2}, t_{3}\right)\right] \rightarrow^{+} D\left[F\left(t_{1} \sigma, t_{2} \sigma, t_{3} \sigma\right)\right]$. Due to minimality not all steps take place inside $t_{1}, t_{2}, t_{3}$. Moreover, at least one of the steps must affect the context $D$ for otherwise we would have $F\left(t_{1}, t_{2}, t_{3}\right) \rightarrow^{+} F\left(t_{1} \sigma, t_{2} \sigma, t_{3} \sigma\right)$ with only applications of the rules $F(\alpha(x), \beta(y), z) \rightarrow F(x, y, z)$ at root positions, but then the size of the maximal topmost part of $t_{1}$ consisting entirely of symbols in $\Gamma$ must exceed that of $t_{1} \sigma$, which is clearly impossible. Hence we obtain a 
non-empty reduction $D[c] \rightarrow^{+} D[c]$ in the TRS consisting of the three rules $h(c) \rightarrow g(c), h(g(x)) \rightarrow g(h(x))$, and $f(g(x)) \rightarrow f(h(h(x)))$. Considering size and observing that the first two rules are terminating yields a contradiction.

Proposition 18. The TRS $\mathcal{S}_{2}(P)$ is terminating if and only if $P$ admits no solution.

Proof. Let $\gamma \in \Gamma^{+}$be a solution for $P$. Write $t=F(\gamma(c), \gamma(c), \gamma(c))$. For every $i>0$ we have the $\mathcal{S}_{2}(P)$-reduction

$$
\begin{aligned}
f\left(h^{i}(t)\right) & =f\left(h^{i}(F(\gamma(c), \gamma(c), \gamma(c)))\right) \rightarrow^{+} f\left(h^{i}(F(c, c, \gamma(c)))\right) \\
& \rightarrow f\left(h^{i-1}(g(t))\right) \rightarrow^{*} f\left(g\left(h^{i-1}(t)\right)\right) \rightarrow f\left(h^{i+1}(t)\right),
\end{aligned}
$$

easily extending to an infinite reduction. Conversely, assume $\mathcal{S}_{2}(P)$ admits an infinite reduction. Erasing all occurrences of $f, g$, and $h$ yields an infinite $\mathcal{R}(P)$ reduction. According to Prop. 14, $P$ admits a solution.

\section{$3.3 \quad \mathrm{NSE} \Rightarrow \mathrm{SN}$}

Let

$$
\mathcal{S}_{3}(P)=\left\{\begin{aligned}
F(c, c, a(z)) & \rightarrow G(a(z), a(z), a(z))) & & \text { for all } a \in \Gamma \\
G(\alpha(x), \beta(y), z) & \rightarrow G(x, y, z) & & \text { for all }(\alpha, \beta) \in P \\
G(c, c, z) & \rightarrow F(h(c), c, z) & &
\end{aligned}\right.
$$

Proposition 19. The TRS $\mathcal{S}_{3}(P)$ is terminating for every PCP instance $P$.

Proof. We apply semantic labelling as described in [21]. As model $\mathcal{M}$ we choose two elements 0 , 1 , with interpretations $F_{\mathcal{M}}(x, y, z)=G_{\mathcal{M}}(x, y, z)=a_{\mathcal{M}}(x)=$ $c_{\mathcal{M}}=1$ and $h_{\mathcal{M}}(x)=0$ for all $x, y, z \in\{0,1\}$ and $a \in \Gamma$. Since all left and right-hand sides of the rules are equal to 1 in this interpretation, $\mathcal{M}$ is indeed a model for $\mathcal{S}_{3}(P)$. Using this model we label the symbol $F$ by the value of its first argument, yielding the labelled system

$$
\mathcal{S}_{3}^{\prime}(P)=\left\{\begin{aligned}
F_{1}(c, c, a(z)) & \rightarrow G(a(z), a(z), a(z)) & & \text { for all } a \in \Gamma \\
G(\alpha(x), \beta(y), z) & \rightarrow G(x, y, z) & & \text { for all }(\alpha, \beta) \in P \\
G(c, c, z) & \rightarrow F_{0}(h(c), c, z) & &
\end{aligned}\right.
$$

The main result of semantic labelling states that $\mathcal{S}_{3}(P)$ is terminating if and only if $\mathcal{S}_{3}^{\prime}(P)$ is terminating. The latter holds by recursive path order: choose $F_{1}>G>F_{0}>h$.

Proposition 20. The TRS $\mathcal{S}_{3}(P)$ is self-embedding if and only if $P$ admits a solution.

Proof. Let $\gamma \in \Gamma^{+}$be a solution for $P$. Then we have the $\mathcal{S}_{3}(P)$-reduction

$$
F(c, c, \gamma(c)) \rightarrow G(\gamma(c), \gamma(c), \gamma(c)) \rightarrow^{+} G(c, c, \gamma(c)) \rightarrow F(h(c), c, \gamma(c)) .
$$


Since $F(h(c), c, \gamma(c)) \rightarrow{ }_{\mathcal{E} \text { mb }} F(c, c, \gamma(c)), \mathcal{S}_{3}(P)$ is self-embedding. Conversely, assume $\mathcal{S}_{3}(P)$ is self-embedding. Let $\psi(t)$ denote the maximal nesting of $F$ and $G$ symbols in a term $t$ :

$$
\begin{aligned}
\psi(x) & =0 & & \text { for } x \in \mathcal{X} \\
\psi(a(t)) & =\psi(t) & & \text { for } a \in \Gamma \\
\psi(c) & =0 & & \psi\left(F\left(t_{1}, t_{2}, t_{3}\right)\right)=1+\max \left\{\psi\left(t_{1}\right), \psi\left(t_{2}\right), \psi\left(t_{3}\right)\right\} \\
\psi(h(t)) & =\psi(t) & & \psi\left(G\left(t_{1}, t_{2}, t_{3}\right)\right)=1+\max \left\{\psi\left(t_{1}\right), \psi\left(t_{2}\right), \psi\left(t_{3}\right)\right\}
\end{aligned}
$$

Obviously, $t \rightarrow_{\mathcal{S}_{3}(P)} u$ implies $\psi(t)=\psi(u)$. Let $t \rightarrow_{\mathcal{S}_{3}(P)}^{+} u \rightarrow_{\mathcal{E}_{\mathrm{mb}}}^{*} t$ be such that $\psi(t)$ is minimal. We may assume that the topmost symbol of $t$ is either (i) $F$ or (ii) $G$. Since $\psi(t)=\psi(u)$ and $u \rightarrow_{\mathcal{E} \mathrm{mb}}^{*} t$ we conclude that the root symbols of $t$ and $u$ coincide. Moreover, by our minimality assumption, there is at least one reduction step in $t \rightarrow_{\mathcal{S}_{3}(P)}^{+} u$ at the root position. First we consider case (i). The $\mathcal{S}_{3}(P)$-reduction from $t$ to $u$ must start as $t=F\left(c, c, a\left(t_{1}\right)\right) \rightarrow^{*}$ $F\left(c, c, a\left(t_{2}\right)\right) \rightarrow G\left(a\left(t_{2}\right), a\left(t_{2}\right), a\left(t_{2}\right)\right)$, where $t_{1} \rightarrow^{*} t_{2}$, for otherwise there would be no reduction step at a root position. Since the root symbols of $t$ and $u$ coincide, it further follows that $G\left(a\left(t_{2}\right), a\left(t_{2}\right), a\left(t_{2}\right)\right) \rightarrow^{+} G\left(c, c, t_{3}\right) \rightarrow F\left(h(c), c, t_{3}\right) \rightarrow^{*}$ $F\left(h(c), c, t_{4}\right)=u$. Consequently, $a\left(t_{2}\right)$ is a solution for $P$. Next we consider case (ii). Write $t=G\left(t_{1}, t_{2}, t_{3}\right)$ and $u=G\left(u_{1}, u_{2}, u_{3}\right)$. Note that the rule $G(c, c, z) \rightarrow$ $F(h(c), c, z)$ is not applicable at root positions in the $\mathcal{S}_{3}(P)$-reduction $(*)$ from $t$ to $u$ because for no term $t^{\prime}$ there is an $\mathcal{S}_{3}(P)$-reduction from $F\left(h(c), c, t^{\prime}\right)$ to $u$. Hence only rules of the form $G(\alpha(x), \beta(y), z) \rightarrow G(x, y, z)$ are applicable at root positions in $(*)$ and thus the root symbol of every term in $(*)$ is $G$. For every reduction step $G\left(t_{1}^{\prime}, t_{2}^{\prime}, t_{3}^{\prime}\right) \rightarrow G\left(u_{1}^{\prime}, u_{2}^{\prime}, u_{3}^{\prime}\right)$ in (*) we have either (1) $t_{1}^{\prime}=u_{1}^{\prime}$ if the reduction took place in $t_{2}^{\prime}$ or $t_{3}^{\prime}$, (2) $t_{1}^{\prime} \rightarrow_{\mathcal{S}_{3}(P)} u_{1}^{\prime}$ if the reduction took place in $t_{1}^{\prime}$, or (3) $t_{1}^{\prime} \triangleright u_{1}^{\prime}$ (i.e., $u_{1}^{\prime}$ is a proper subterm of $t_{1}^{\prime}$ ) if the reduction took place at the root. By assumption alternative (3) occurs at least once. Using the well-known facts that $\triangleright \cdot \rightarrow_{\mathcal{S}_{3}(P)} \subseteq \rightarrow_{\mathcal{S}_{3}(P)} \cdot \triangleright$ and $\triangleright \cdot \triangleright \subseteq \triangleright$ it follows that $t_{1} \rightarrow_{\mathcal{S}_{3}(P)}^{*} \cdot \triangleright u_{1}$. Because $\psi(t)=\psi(u)$, there are no reduction steps at root positions in $u \rightarrow_{\mathcal{E}_{\mathrm{mb}}^{*}}^{*} t$. Hence $u_{1} \rightarrow_{\mathcal{E} \mathrm{mb}}^{*} t_{1}$. Combining this with $t_{1} \rightarrow_{\mathcal{S}_{3}(P)}^{*} \cdot \triangleright u_{1}$ yields $t_{1} \rightarrow_{\mathcal{S}_{3}(P)}^{+} \cdot \rightarrow_{\mathcal{E}_{\mathrm{mb}}^{*}}^{*} t_{1}$. However, $\psi\left(t_{1}\right)<\psi(t)$, contradicting the minimality of $\psi(t)$. We conclude that case (ii) is impossible.

\section{$3.4 \mathrm{ST} \Rightarrow \mathrm{NSE}$}

Let $\mathcal{S}_{4}(P)$ be the TRS

$$
\left\{\begin{array}{rlrl}
F(x, \bar{a}(y), x, \bar{a}(y)) & \rightarrow F(h(a(x)), h(y), a(x), y) & & \text { for all } a \in \Gamma \\
F(\alpha(x), y, \beta(z), w) & \rightarrow F(h(x), h(\bar{\alpha}(y)), z, \bar{\beta}(w)) & \text { for all }(\alpha, \beta) \in P
\end{array}\right.
$$

Proposition 21. The TRS $\mathcal{S}_{4}(P)$ is non-self-embedding for every PCP instance $P$.

Proof. For a term $t$, let $\|t\|$ denote the number of $F, a$, and $\bar{a}$ symbols in $t$. Clearly $\|t\|=\|u\|$ for every reduction step $t \rightarrow_{\mathcal{S}_{4}(P)} u$. For a proof by contradiction, 
assume a self-embedding reduction $t \rightarrow_{\mathcal{S}_{4}(P)}^{+} u \rightarrow_{\mathcal{E} \text { mb }}^{*} t(*)$ exists. Since $\|t\|=$ $\|u\|$, in $u \rightarrow_{\mathcal{E}_{\mathrm{mb}}}^{*} t$ only the rule $h(x) \rightarrow x$ is applied. Just as in the proof of Prop. 20 we may assume that there is at least one reduction step in $t \rightarrow_{\mathcal{S}_{4}(P)}^{+} u$ at the root position. Hence we may write $(*)$ as

$$
t=\underbrace{F\left(t_{1}, t_{2}, t_{3}, t_{4}\right) \rightarrow^{*} F\left(u_{1}, u_{2}, u_{3}, u_{4}\right)} \rightarrow \underbrace{F\left(h\left(v_{1}\right), h\left(v_{2}\right), v_{3}, v_{4}\right) \rightarrow^{*} u \rightarrow^{*} t}
$$

where in the underbraced parts no steps take place at root positions. (Note that no term of the form $F\left(h\left(v_{1}\right), h\left(v_{2}\right), v_{3}, v_{4}\right)$ is a redex.) We obtain $\left\|t_{1}\right\|=\left\|u_{1}\right\| \neq$ $\left\|h\left(v_{1}\right)\right\|=\left\|t_{1}\right\|$, which is a contradiction.

Proposition 22. The TRS $\mathcal{S}_{4}(P)$ is simply terminating if and only if $P$ admits no solution.

Proof. According to Prop. 14 it is sufficient to show that $\mathcal{S}_{4}(P)$ is simply terminating if and only if $\mathcal{S}(P)$ is terminating. Suppose $\mathcal{S}(P)$ is non-terminating. Since $\rightarrow_{\mathcal{S}(P)} \subseteq \rightarrow_{\mathcal{S}_{4}(P) \cup \mathcal{E} \mathrm{mb}}^{+}$, also $\mathcal{S}_{4}(P) \cup \mathcal{E} \mathrm{mb}$ is non-terminating and hence $\mathcal{S}_{4}(P)$ is not simply terminating. Conversely, assume $\mathcal{S}(P)$ is terminating. Since $\mathcal{S}(P)$ is length-preserving it is simply terminating and thus admits a compatible simple monotone algebra $(A,>)$. By defining $h_{A}(x)=x$ for $x \in A$ this becomes a simple monotone algebra compatible with $\mathcal{S}_{4}(P)$, hence $\mathcal{S}_{4}(P)$ is simply terminating.

\section{$3.5 \mathrm{TT} \Rightarrow \mathrm{ST}$}

Let

$$
\mathcal{S}_{5}(P)=\left\{\begin{array}{cl}
F(x, \bar{a}(y), x, \bar{a}(y)) \rightarrow F(\hat{a}(x), y, \tilde{a}(x), y) & \text { for all } a \in \Gamma \\
F(\hat{a}(x), y, \hat{a}(x), y) \rightarrow F(a(x), y, a(x), y) & \text { for all } a \in \Gamma \\
F(\tilde{a}(x), y, \tilde{a}(x), y) \rightarrow F(a(x), y, a(x), y) & \text { for all } a \in \Gamma \\
F(\alpha(x), y, \beta(z), w) \rightarrow F(x, \bar{\alpha}(y), z, \bar{\beta}(w)) & \text { for all }(\alpha, \beta) \in P
\end{array}\right.
$$

Proposition 23. The TRS $\mathcal{S}_{5}(P)$ is simply terminating for every $P C P$ instance $P$.

Proof. Since $\mathcal{S}_{5}(P)$ is length-preserving it suffices to prove termination. We apply semantic labelling. As model $\mathcal{M}$ we choose two elements 0,1 , with interpretations $F_{\mathcal{M}}(x, y, z, w)=a_{\mathcal{M}}(x)=\bar{a}_{\mathcal{M}}(x)=\hat{a}_{\mathcal{M}}(x)=0$ and $\tilde{a}_{\mathcal{M}}(x)=1$ for all $x, y, z, w \in\{0,1\}$ and $a \in \Gamma$. Since all left and right-hand sides of the rules are equal to 0 in this interpretation, $\mathcal{M}$ is indeed a model for $\mathcal{S}_{5}(P)$. Using this model we label the symbol $F$ as follows: it is labelled by 1 if the interpretations of the first and the third argument are equal, and by 0 otherwise. This yields the labelled system

$$
\mathcal{S}_{5}^{\prime}(P)=\left\{\begin{array}{cl}
F_{1}(x, \bar{a}(y), x, \bar{a}(y)) \rightarrow F_{0}(\hat{a}(x), y, \tilde{a}(x), y) & \text { for all } a \in \Gamma \\
F_{1}(\hat{a}(x), y, \hat{a}(x), y) \rightarrow F_{1}(a(x), y, a(x), y) & \text { for all } a \in \Gamma \\
F_{1}(\tilde{a}(x), y, \tilde{a}(x), y) \rightarrow F_{1}(a(x), y, a(x), y) & \text { for all } a \in \Gamma \\
F_{1}(\alpha(x), y, \beta(z), w) \rightarrow F_{1}(x, \bar{\alpha}(y), z, \bar{\beta}(w)) & \text { for all }(\alpha, \beta) \in P \\
F_{1}(\alpha(x), y, \beta(z), w) \rightarrow F_{0}(x, \bar{\alpha}(y), z, \bar{\beta}(w)) & \text { for all }(\alpha, \beta) \in P
\end{array}\right.
$$


From [21] we know that $\mathcal{S}_{5}(P)$ is terminating if and only if $\mathcal{S}_{5}^{\prime}(P)$ is terminating. The latter holds by lexicographic path order: choose $F_{1}>F_{0}>\hat{a}>\tilde{a}>a>\bar{a}$ for all $a \in \Gamma$ and compare the arguments of $F_{1}$ from left to right.

Proposition 24. The TRS $\mathcal{S}_{5}(P)$ is totally terminating if and only if $P$ admits no solution.

Proof. Assume $P$ admits no solution. According to [22] the TRS $\mathcal{S}(P)$ is totally terminating, hence admits a compatible well-founded monotone algebra $(A,>)$ with $>$ a total order on $A$. We define the well-founded monotone algebra $(B, \succ)$ by $B=A \times \mathbb{N},(x, n) \succ\left(x^{\prime}, n^{\prime}\right)$ if and only if $x>x^{\prime}$ or $x=x^{\prime}$ and $n>n^{\prime}$, and interpretations

$$
\begin{aligned}
& F_{B}\left(\left(x_{1}, n_{1}\right),\left(x_{2}, n_{2}\right),\left(x_{3}, n_{3}\right),\left(x_{4}, n_{4}\right)\right)=\left(F_{A}\left(x_{1}, x_{2}, x_{3}, x_{4}\right), n_{1}+n_{2}+n_{3}+n_{4}\right) \\
& a_{B}((x, n))=\left(a_{A}(x), n\right) \\
& \bar{a}_{B}((x, n))=\left(\bar{a}_{A}(x), n\right) \\
& \hat{a}_{B}((x, n))=\tilde{a}_{B}((x, n))=\left(a_{A}(x), n+1\right) \\
& c_{B}=\left(c_{A}, 0\right)
\end{aligned}
$$

One easily verifies that $(B, \succ)$ is a well-founded monotone algebra compatible with $\mathcal{S}_{5}(P)$. Since $\succ$ is a total order on $B, \mathcal{S}_{5}(P)$ is totally terminating. Conversely, assume that $\gamma \in \Gamma^{+}$is a solution for $P$. For a proof by contradiction, suppose that $\mathcal{S}_{5}(P)$ is totally terminating. Then $\mathcal{S}_{5}(P)$ admits a compatible monotonic well-founded total order $>$ on ground terms. Let $t, u$ be arbitrary ground terms (remember that a constant $c \in \mathcal{F}$ is assumed) and $a \in \Gamma$. If $\hat{a}(t)>\tilde{a}(t)$ then

$$
F(\hat{a}(t), u, \tilde{a}(t), u)>F(\tilde{a}(t), u, \tilde{a}(t), u)>F(a(t), u, a(t), u),
$$

otherwise $\tilde{a}(t)>\hat{a}(t)$ and

$$
F(\hat{a}(t), u, \tilde{a}(t), u)>F(\hat{a}(t), u, \hat{a}(t), u)>F(a(t), u, a(t), u) .
$$

Hence for all ground terms $t, u$ and all $a \in \Gamma$ we obtain $F(\hat{a}(t), u, \tilde{a}(t), u)>$ $F(a(t), u, a(t), u)$. Using this result and the compatibility of $\mathcal{S}_{5}(P)$ and $>$ yields

$$
\begin{aligned}
& F(\gamma(c), c, \gamma(c), c)>\cdots>F(c, \bar{\gamma}(c), c, \bar{\gamma}(c)) \\
& \quad=F\left(c, \bar{a}\left(\overline{\gamma_{1}}(c)\right), c, \bar{a}\left(\overline{\gamma_{1}}(c)\right)\right)>F\left(\hat{a}(c), \overline{\gamma_{1}}(c), \tilde{a}(c), \overline{\gamma_{1}}(c)\right) \\
& \quad>F\left(a(c), \overline{\gamma_{1}}(c), a(c), \overline{\gamma_{1}}(c)\right)>\cdots>F(\gamma(c), c, \gamma(c), c),
\end{aligned}
$$

where $\gamma$ is written as $\gamma_{1} a$, contradicting the irreflexivity of $>$.

\section{$3.6 \quad \omega \mathrm{T} \Rightarrow \mathrm{TT}$}

Let $\mathcal{S}_{6}(P)$ be the TRS

$$
\left\{\begin{aligned}
F(c, \bar{a}(y), c, \bar{a}(w), u) & \rightarrow G(a(c), y, a(c), w, h(h(u))) & & \text { for all } a \in \Gamma \\
G(x, \bar{a}(y), z, \bar{a}(w), u) & \rightarrow G(a(x), y, a(z), w, u) & & \text { for all } a \in \Gamma \\
h(G(\alpha(x), c, \beta(z), c, u)) & \rightarrow F(x, \bar{\alpha}(c), z, \bar{\beta}(c), u) & & \text { for all }(\alpha, \beta) \in P \\
F(\alpha(x), y, \beta(z), w, u) & \rightarrow F(x, \bar{\alpha}(y), z, \bar{\beta}(w), u) & & \text { for all }(\alpha, \beta) \in P
\end{aligned}\right.
$$


Proposition 25 [6]. The TRS $\mathcal{S}_{6}(P)$ is totally terminating for every PCP instance $P$.

Proof. We use the Knuth-Bendix order ([11]) where $h$ is assigned weight 0 , and every other function symbol weight 1 . For the precedence we choose $h>F>$ $G>a>\bar{a}>c$ for all $a \in \Gamma$. Note that $h$ satisfies the constraint the KnuthBendix order requires, namely that every function symbol of weight 0 is unary and greatest in precedence. We take lexicographic status for each function symbol, $F$ left-to-right and $G$ right-to-left. The induced Knuth-Bendix order orients each rule from left to right. By a result of Ferreira [4, Thm. 4.47] the TRS $\mathcal{S}_{6}(P)$ is not only terminating, but even totally terminating.

Proposition 26 [6]. The TRS $\mathcal{S}_{6}(P)$ is $\omega$-terminating if and only if $P$ admits no solution.

Proof. Let $\gamma \in \Gamma^{+}$be a solution for $P$. Then we have for all ground terms $t$ an $\mathcal{S}_{6}(P)$-reduction

$$
\begin{aligned}
h(G(\gamma(c), c, \gamma(c), c, t)) & \rightarrow^{+} F(c, \bar{\gamma}(c), c, \bar{\gamma}(c), c, t) \\
& \rightarrow^{+} G(\gamma(c), c, \gamma(c), c, h(h(t))) .
\end{aligned}
$$

For a proof by contradiction, assume that $\mathcal{S}_{6}(P)$ is $\omega$-terminating, with compatible well-founded monotone algebra $(A,>)$ where $A=\mathbb{N}$. Define $f_{A}(t)=$ $G_{A}\left([\gamma(c)], c_{A},[\gamma(c)], c_{A}, t\right)$. The above reduction proves $\omega$-termination of the single rule $h(f(x)) \rightarrow f(h(h(x)))$, contradicting [20, Prop. 11]. Hence $\mathcal{S}_{6}(P)$ is not $\omega$-terminating.

This leaves to prove that $\mathcal{S}_{6}(P)$ is $\omega$-terminating if $P$ has no solution. Here we sketch the proof, for more details we refer to [6]. For a ground term $t$, let $\|t\|$ denote the number of barred and unbarred letters in $t$ not below an $F$, $G$, or $h$ symbol. An analysis of reduction patterns shows that any reduction starting from a term of the shape $h^{k}(G(p, q, r, s, t))$ or $h^{k}(F(p, q, r, s, t))$ with at least $3 \cdot \min \{\|p\|+\|q\|,\|r\|+\|s\|\}$ steps at the topmost $F$ or $G$ symbol, gives rise to a solution for $P$. We define $\operatorname{len}_{F}(p, q, r, s)$ to be the maximum number of reduction steps at the topmost $F$ or $G$ symbol, starting from a term of the shape $h^{k}(F(p, q, r, s, t))$. Similarly we define $\operatorname{len}_{G}(p, q, r, s)$ for starting terms of the shape $h^{k}(G(p, q, r, s, t))$. We define auxiliary functions on $\mathbb{N}_{+}$as follows:

$$
\begin{aligned}
& \operatorname{conc}(x, y)=10^{\lfloor\log (y)\rfloor+1} \cdot x+y \\
& \operatorname{revc}(x, y)=\operatorname{conc}(\operatorname{conc}(x, y), \underbrace{4 \ldots 4}_{\lfloor\log (x)\rfloor+1}) \\
& \operatorname{digits} \\
& \operatorname{bound}(x, y, z, w)=3 \cdot \min \{\lfloor\log (\operatorname{revc}(x, y))\rfloor,\lfloor\log (\operatorname{revc}(z, w)\rfloor\}
\end{aligned}
$$


Next an auxiliary function $\pi: \mathbb{N}_{+} \rightarrow \mathcal{T}(\mathcal{F})$ is defined recursively as follows:

$$
\pi(x)= \begin{cases}0\left(\pi\left(x^{\prime}\right)\right) & \text { if } x=10 x^{\prime}+2 \\ 1\left(\pi\left(x^{\prime}\right)\right) & \text { if } x=10 x^{\prime}+3 \\ \overline{0}\left(\pi\left(x^{\prime}\right)\right) & \text { if } x=10 \cdot \operatorname{conc}\left(2, x^{\prime}\right)+4 \\ \overline{1}\left(\pi\left(x^{\prime}\right)\right) & \text { if } x=10 \cdot \operatorname{conc}\left(3, x^{\prime}\right)+4 \\ c & \text { if } x=1 \\ h(c) & \text { otherwise }\end{cases}
$$

Now we define the well-founded monotone algebra $(A,>)$ with $A=\mathbb{N}_{+}$:

$$
\begin{array}{rlr}
c_{A} & =1 & \\
0_{A}(x) & =10 x+2 \quad \overline{0}_{A}(x)=10 \cdot \operatorname{conc}(2, x)+4 \\
1_{A}(x) & =10 x+3 \quad \overline{1}_{A}(x)=10 \cdot \operatorname{conc}(3, x)+4 \\
h_{A}(x) & =10 x+5 \\
F_{A}(x, y, z, w, u) & =6+10 u \cdot 156^{\operatorname{len}_{F}(\pi(x), \pi(y), \pi(z), \pi(w))+1+B} \\
G_{A}(x, y, z, w, u) & =7+10 u \cdot 156^{\operatorname{len}_{G}(\pi(x), \pi(y), \pi(z), \pi(w))+1+B}
\end{array}
$$

where $B=(\operatorname{bound}(x, y, z, w)+1)(\operatorname{revc}(x, y)+\operatorname{revc}(z, w))$. The definition of $\pi$ expresses the fact that from $[t]$ one can reconstruct the sequence of barred and unbarred letters of $t$. This information is essential to determine the estimated maximal topmost reduction length of $F$ and $G$ terms. Formally, structural induction on $\zeta$ shows:

for all strings $\zeta$ of barred and unbarred letters and for all ground terms $t$ and $t^{\prime}, \pi([t])=\zeta\left(t^{\prime}\right)$ is equivalent to the existence of a ground term $t^{\prime \prime}$ such that $t=\zeta\left(t^{\prime \prime}\right)$ and $\pi\left(\left[t^{\prime \prime}\right]\right)=t^{\prime}$.

Structural induction on $\alpha$ proves that

$$
\operatorname{revc}([\theta](\alpha(x)),[\theta](y))=\operatorname{revc}([\theta](x),[\theta](\bar{\alpha}(y)))
$$

for $\alpha \in \Gamma^{*}, \theta: \mathcal{X} \rightarrow \mathbb{N}_{+}$, and $x, y \in \mathcal{X}$. Now one can show that $[l \sigma]>[r \sigma]$ for any ground instance $l \sigma \rightarrow r \sigma$ of a rule in $\mathcal{S}_{6}(P)$. The central step in this proof is $156 \cdot[t]>155 \cdot[t]=[h(h(t))]$ for the first type of rules. The functions $0_{A}, 1_{A}$, and $h_{A}$ are trivially monotonic. Observing that conc and revc are monotonic, and that bound is weakly monotonic, in every argument, one obtains that $\overline{0}_{A}$, $\overline{1}_{A}$ are monotonic as well. To establish that $F_{A}$ and $G_{A}$ are monotonic in all arguments, one proves first that if $P$ has no solution then for all $x, y, z, w \in \mathbb{N}_{+}$

$$
\operatorname{len}_{F}(\pi(x), \pi(y), \pi(z), \pi(w))<\operatorname{bound}(x, y, z, w)
$$

and

$$
\operatorname{len}_{G}(\pi(x), \pi(y), \pi(z), \pi(w))<\operatorname{bound}(x, y, z, w) .
$$


Obviously $F_{A}$ and $G_{A}$ are monotonic in their last argument. For the other arguments, let $x \leq x^{\prime}, y \leq y^{\prime}, z \leq z^{\prime}, w \leq w^{\prime}$ where at least one of these inequalities is strict, and prove that $\log _{156}\left\lfloor\frac{F_{A}(x, y, z, w, u)}{10 u}\right\rfloor<\log _{156}\left\lfloor\frac{F_{A}\left(x^{\prime}, y^{\prime}, z^{\prime}, w^{\prime}, u^{\prime}\right)}{10 u}\right\rfloor$. So indeed we arrive at a compatible well-founded monotone algebra corresponding to the positive integers $\mathbb{N}_{+}$, which is order isomorphic to $\mathbb{N}$, hence $\mathcal{S}_{6}(P)$ is $\omega$ terminating. This completes the proof.

\subsection{WN and CR}

None of the TRSs $\mathcal{R}(P), \mathcal{S}(P), \mathcal{S}_{1}(P)$ to $\mathcal{S}_{6}(P)$ is confluent for arbitrary PCP instances. This can be repaired by adding appropriate rewrite rules of the shape $F(x, y, z, \ldots) \rightarrow c, G(x, y, z, \ldots) \rightarrow c, f(c) \rightarrow c, g(c) \rightarrow c$, and $h(c) \rightarrow c$ without affecting any of our propositions. This has the additional benefit of making the TRSs $\mathcal{S}_{1}(P)$ and $\mathcal{S}_{2}(P)$ weakly normalizing. (Note that $\mathcal{S}_{3}(P)$ to $\mathcal{S}_{6}(P)$ are always terminating.) Consequently all of our results hold for confluent weakly normalizing TRSs.

In particular, the union of $\mathcal{R}(P)$ and the rule $F(x, y, z) \rightarrow c$ is easily seen to be weakly normalizing while it is terminating if and only if $P$ admits no solution as in Prop. 14. This proves relative undecidability of the implication SN $\Rightarrow$ WN.

\section{Conclusions}

For most of the implications in the confluence and termination hierarchies we proved relative undecidability; only for polynomial termination the question of (relative) undecidability is still open.

One can wonder whether similar results hold for TRSs consisting of single rules. Undecidability of termination ([2]), and non-self-embeddingness and simple termination ([15]) of single rules was already known. For the lower four implications in the termination hierarchy indeed relative undecidability for single (even orthogonal) rules can be proved; this work is still in progress.

\section{References}

1. A.-C. Caron, Linear Bounded Automata and Rewrite Systems: Influence of Initial Configuration on Decision Properties, Proceedings of the 15th Colloquium on Trees in Algebra and Programming, Lecture Notes in Computer Science 493 (1991) 7489.

2. M. Dauchet, Simulation of Turing Machines by a Regular Rewrite Rule, Theoretical Computer Science 103 (1992) 109-120.

3. N. Dershowitz and J.-P. Jouannaud, Rewrite Systems, in: Handbook of Theoretical Computer Science, Vol. B (ed. J. van Leeuwen), North-Holland (1990) 243-320.

4. M.C.F. Ferreira, Termination of Term Rewriting: Well-foundedness, Totality, and Transformations, PhD thesis, University of Utrecht, 1995.

5. M.C.F. Ferreira and H. Zantema, Total Termination of Term Rewriting, Applicable Algebra in Engineering, Communication and Computing 7 (1996) 133-162. 
6. A. Geser, Omega-termination is Undecidable for Totally Terminating Term Rewriting Systems, Technical Report MIP-9608, Universität Passau (1996).

7. G. Huet and D. Lankford, On the Uniform Halting Problem for Term Rewriting Systems, report 283, INRIA (1978).

8. G. Huet, Confluent Reductions: Abstract Properties ans Applications to Term Rewriting Systems, Journal of the ACM 27 (1980) 797-821.

9. G. Huet and D.C. Oppen, Equations and Rewrite Rules: A Survey, in: Formal Language Theory: Perspectives and Open Problems (ed. R. Book), Academic Press (1980) 349-405.

10. J.W. Klop, Term Rewriting Systems, in: Handbook of Logic in Computer Science (eds. S. Abramski, D. Gabbay and T. Maibaum), Volume 2, Oxford University Press (1992) 1-116.

11. D.E. Knuth and P.B. Bendix, Simple Word Problems in Universal Algebras, in: Computational Problems in Abstract Algebra (ed. J. Leech), Pergamon Press (1970) 263-297.

12. M. Kurihara and A. Ohuchi, Modularity of Simple Termination of Term Rewriting Systems, Journal of IPS Japan 31 (1990) 633-642.

13. P. Lescanne, On Termination of One Rule Rewrite Systems, Theoretical Computer Science 132 (1994) 395-401.

14. Y. Matiyasevich and G. Senizergues, Decision Problems for Semi-Thue Systems with a Few Rules. Proceedings of the 11th IEEE Annual Symposium on Logic in Computer Science, New Brunswick, pp. 523-531, 1996.

15. A. Middeldorp and B. Gramlich, Simple Termination is Difficult, Applicable Algebra in Engineering, Communication and Computing 6 (1995) 115-128.

16. A. Middeldorp and H. Zantema, Simple Termination Revisited, Proceedings of the 12th International Conference on Automated Deduction, Nancy, Lecture Notes in Artificial Intelligence 814 (1994) 451-465. Extended version to appear as Simple Termination of Rewrite Systems in Theoretical Computer Science 175 (1997).

17. D.A. Plaisted, The Undecidability of Self-Embedding for Term Rewriting Systems, Information Processing Letters 20 (1985) 61-64.

18. E. Post, A Variant of a Recursively Unsolvable Problem, Bulletin of the American Mathematical Society 52 (1946) 264-268.

19. A. Rubio, Extension Orderings, Proceedings of the 22nd International Colloquium on Automata, Languages and Programming, Szeged, Lecture Notes in Computer Science 944 (1995) 511-522.

20. H. Zantema, Termination of Term Rewriting: Interpretation and Type Elimination, Journal of Symbolic Computation 17 (1994) 23-50.

21. H. Zantema, Termination of Term Rewriting by Semantic Labelling, Fundamenta Informaticae 24 (1995) 89-105.

22. H. Zantema, Total Termination of Term Rewriting is Undecidable, Journal of Symbolic Computation 20 (1995) 43-60.

23. H. Zantema and A. Geser, Non-Looping Rewriting, Utrecht University, Department of Computer Science, report UU-CS-1996-03 (1996). 